\title{
Medizintheoretische und wissenschaftshistorische Perspektiven einer Revision der Materia medica homoeopathica*
}

\author{
Josef M. Schmidt \\ Institut für Geschichte der Medizin, Ludwig-Maximilians-Universität, München, Deutschland
}

\section{Schlüsselwörter}

Homöopathie - Medizintheorie · Wissenschaftsgeschichte · Materia medica homoeopathica - Samuel Hahnemann . Heilkunst · Friedrich Nietzsche · Postmoderne

\section{Zusammenfassung}

In der Homöopathie ist das, was an Patienten und Arzneimittelprüfern beobachtet wird, nicht trivial. Symptome sind hier keine Messwerte, sondern ihre Wahrnehmung, Aufzeichnung und praktische Verwertung sind abhängig von der jeweils zugrunde gelegten (homöopathischen) Theorie. Eine Revision der Materia medica homoeopathica braucht daher vordringlich eine Verständigung über eine tragfähige und konsensfähige kritische Theorie der Homöopathie. Als Grundlinien bzw. Elemente einer solchen werden unter anderem vorgeschlagen: disziplinäres Selbstverständnis als praktische Wissenschaft sui generis, medizinhistorisches Bewusstsein der Einbettung in sozioökonomische, politische und kulturelle Rahmenbedingungen, medizintheoretische Einschätzung des Stellenwertes naturwissenschaftlich-statistischer Methoden, philosophische Bewertung der traditionellen metaphysischen Dogmen und wissenschaftstheoretische Auseinandersetzung mit den modernen Geistes-, Sozial- und Naturwissenschaften. Die zentrale Argumentation stellt - angelehnt an Nietzsches genealogische Wissenschaftskritik - unkritischen Wissenschaftsoptimismus infrage und plädiert für eine Höherstufung des Status des (Heil-)Künstlers, der immerhin in jedem Einzelfall aufs Neue zu beurteilen hat, wann, wo und unter welchen Umständen welche wissenschaftliche Methode einzusetzen sei. Unter Verabschiedung dogmatischer Wahrheitsbegriffe und Bekräftigung des instrumentellen Charakters homöopathischer Theorien könnte die Homöopathie die Herausforderungen der Postmoderne meistern - als semiotisches Kombinationssystem, mit dem Primat der Heilkunst über dem Anspruch auf Wissenschaftlichkeit.

\author{
Keywords \\ Homeopathy - Theory of medicine $\cdot$ History of science $\cdot$ Materia \\ medica homoeopathica - Samuel Hahnemann - Art of healing . \\ Friedrich Nietzsche · Postmodernity
}

\section{Summary}

A Revision of the Homeopathic Materia Medica from the Perspective of Theory of Medicine and History of Science

In homeopathy, observing patients and drug provers is not trivial, since symptoms do not merely consist of measured data. In fact, their perception, recording, and practical application depend on the respective underlying (homeopathic) theory. Hence, a revision of the homeopathic materia medica requires a discussion about a sound and reasonable critical theory of homeopathy. As main outlines and elements of such a theory are suggested among others: self-concept as a practical science sui generis, historical consciousness of being embedded in socio-economic, political, and cultural settings, medical assessment of the status of scientific and statistical methods, philosophical valuation of traditional metaphysical doctrines, and theoretical discussion of modern social and natural sciences. Leaning on Nietzsche's genealogic criticism of science, the central argumentation questions uncritical optimism of science and advocates an upgrade of the status of the (healing) artist who, after all, is in charge of judging in every single case anew when, where, and under what circumstances which scientific method needs to be applied. Abandoning dogmatic concepts of truth and embracing the instrumental character of homeopathic theories, homeopathy could meet the challenges of postmodernity - as a semiotic combination system, with primacy of the art of healing above the claim of its scientific character.

\footnotetext{
*Überarbeitete Version eines Vortrags, gehalten anlässlich des

9. Internationalen Köthener Erfahrungsaustauschs am 9.11.2009

im Veranstaltungszentrum Schloss Köthen (Anhalt), Deutschland.
}

$\begin{array}{ll}\text { KARGER } & \text { @ 2010 S. Karger GmbH, Freiburg } \\ \text { Fax +4976145207 14 } & \text { Accessible online at: } \\ \text { Information@Karger.de } & \text { www.karger.com/szg }\end{array}$

PD Dr. med. Dr. phil. Josef M. Schmidt

Institut für Geschichte der Medizin

Ludwig-Maximilians-Universität München

Lessingstrasse 2, 80336 München, Deutschland

j.m.schmidt@lrz.uni-muenchen.de 


\section{Einleitung}

Die Ausübung der Homöopathie ist sicherlich in erster Linie eine Praxis. Gleichwohl bedarf sie, soll dies auf kunstvolle Art geschehen oder gar Skeptiker überzeugen, einer eigenen medizinischen Theorie. Schon ihr Begründer, Samuel Hahnemann (1755-1843), zitierte 1805 Gregor von Nazianz mit den Worten «atelès álogos práxis kaì lógos ápraktos», um zu verdeutlichen, dass «weder theorielose Praxis noch praxislose Theorie zum Ziel führt» [1]. Neben seinem theoretischen Hauptwerk [2-4] hat Hahnemann allerdings eine umfangreiche Arzneimittellehre hinterlassen [5], die so etwas wie einen theorielosen Arzneischatz darstellen soll, einen «Kodex der Natur» bzw. die «reine Sprache der sorgfältig und redlich befragten Natur» [6].

Im Rahmen einer seit Langem anstehenden Revision dieser Materia medica stellen sich grundsätzliche - theoretische - Fragen: Was ist überhaupt ein Symptom, wo fängt es an, wo hört es auf? Gibt es vollständige und unvollständige Symptome? Ist der genaue Wortlaut wichtig oder lässt sich das Wesentliche davon in eine Art Fachsprache übersetzen? Zählen nur die Symptome aus reinen Arzneimittelprüfungen oder auch Berichte über deren Bestätigung in der Praxis (Verifikationen) sowie klinische Beobachtungen (Heilwirkungen)? Wie sind verifizierte Symptome, Charakteristika, Schlüsselsymptome oder Ähnliches zu werten bzw. hervorzuheben (Grade)? Sind sog. Erst- und Nachwirkungen zu unterscheiden? Aus welchen Quellen stammen die Symptome, wie zuverlässig sind diese?

Die Suche nach Kriterien zur Klärung dieser Punkte führt schnell zu theoretischen Fragen noch grundsätzlicherer Art, wie etwa: Ist die Homöopathie prinzipiell gebunden an bestimmte quasidogmatische Aussagen ihres Begründers (wie dies ansonsten für Sekten von Gläubigen typisch ist)? Oder ist sie eine moderne Wissenschaft, die fortschrittsoffen und rational ständig neue Argumente und Theorien entwickelt, prüft, modifiziert und gegebenenfalls auch verwirft? Oder ist sie primär eben eine Praxis, die pragmatisch immer nur das an Erkenntnissen und Empfehlungen verwendet, was sich bei der Behandlung von Patienten bewährt?

An diesem Punkt erhält die traditionelle Haltung der Homöopathen, sich primär als Praktiker zu empfinden, die die Lehre Hahnemanns am individuellen Patienten anwenden, derzeit Rückenwind aus der neueren Entwicklung der Medizintheorie. Gestützt auf Vorarbeiten von Wolfgang Wieland und anderen [7], konstatieren Medizintheoretiker wie Urban Wiesing z.B., dass Medizin keineswegs nur angewandte Naturwissenschaft sei (wie dies bislang überwiegend vertreten wurde), sondern sich als praktische Wissenschaft sui generis begreifen sollte [8] Diese neue/alte Einsicht beruhe auf dem strukturellen Unterschied zwischen naturwissenschaftlichem Wissen, das aus möglichst exakten bzw. statistisch zu verifizierenden (oder falsifizierenden) Allgemeinheiten besteht, und ärztlichem Handeln, das mit der nicht normierbaren, konkreten Entscheidung am einzelnen Patienten zu tun hat - eine Unterscheidung, die letztlich auf Aristoteles zurückgeht $[9,10]$.

Bis hierher könnte der neue pragmatische Umdenkungsprozess in der Theorie der Medizin der Homöopathie sehr gelegen erscheinen, zumal diese sich seit jeher als praktische Wissenschaft verstand [11]. Wenn es allerdings weiter heisst, angesichts der postmodernen Pluralität alternativer heilkundlicher Paradigmen sollten diese - angeblich rein pragmatisch - durch klinische Studien im Sinne von Evidenzbasierter Medizin (EBM) überprüft und aussortiert werden, müssten Homöopathen aufmerken. Hier gewinnt die zunächst rein theoretische Frage, ob sich die Homöopathie als Dogmatik, Wissenschaft oder Praxis definieren sollte, praktische Relevanz und Brisanz. Die zunächst inner-homöopathische Frage weitet sich nun zur existenziellen Herausforderung, die eigene Position im Hinblick auf die Schnittstellen der Homöopathie zum Rest der Welt zu überdenken [12]. Vom Raster der EBM aus geurteilt, hätte jedenfalls ein Heilsystem, das sich nicht wissenschaftlich fassen lässt, seine Existenzberechtigung a priori verwirkt.

\section{Ist die Homöopathie eine Wissenschaft?}

Einer der Faktoren, die das Schicksal der Homöopathie massgeblich bestimmen und für eine Theorie der Homöopathie grösste Bedeutung haben, ist ihr Verhältnis zur Naturwissenschaft. Die spontane Antwort von Homöopathen besteht gewöhnlich in einem klaren Bekenntnis zur Wissenschaftlichkeit, ja, in dem Anspruch, mit der Homöopathie eine wissenschaftliche, wenn nicht gar die wissenschaftliche Medizin zu vertreten [13]. Mit diesem Standpunkt glaubt man sich in bester homöopathischer Gesellschaft. Hatte nicht Hahnemann selbst betont, die Medizin in den Rang einer sicheren Wissenschaft erheben $\mathrm{zu}$ wollen [14], in der es möglich sein sollte, mit «mathematischer» «Zuverlässigkeit» und «Gewissheit» [15] zu heilen? Und gibt es nicht seither eine bis zum heutigen Tag nicht abgerissene Tradition von Beteuerungen der Wissenschaftlichkeit der Homöopathie [16]?

Warum wird die Homöopathie dann aber von der akademischen Medizin nicht anerkannt - und zwar genau mit dem Argument, dass sie die aktuellen Standards von Wissenschaftlichkeit nicht erfüllt? Diese bestünden - methodisch - in dem, was heute EBM genannt wird (randomisierte klinische Studien, statistische Evaluationen, Metaanalysen und Ähnliches), und - inhaltlich - in physikalisch-chemischen, molekularbiologischen und (epi-) genetischen Vorstellungen über die Funktionsweise des 
menschlichen Körpers. Offensichtlich unterscheiden sich die Auffassungen dessen, was man für Wissenschaft hält, beträchtlich.

Die Frage, ob und, wenn ja, inwieweit die Homöopathie als Wissenschaft zu konzipieren, zu praktizieren und weiterzuentwickeln ist, ist aber keine rein akademische Frage, sondern betrifft den Kern sehr realer praktischer Projekte. Je nach zugrunde gelegter Theorie müsste man etwa bei einer Revision der Materia medica ganz unterschiedlich vorgehen. Hielte man sich z.B. streng an die Kriterien der naturwissenschaftlichen Methode, wie etwa Quantifizierbarkeit, Reproduzierbarkeit und Standardisierbarkeit, so dürfte sich wohl kaum ein Symptom in der gesamten Materia medica finden lassen, das diesen Anforderungen exakt entspricht. Welches Prüfungssymptom ist schon quantifizierbar oder unter gleichen Versuchsbedingungen hundertprozentig reproduzierbar?

Allenfalls könnte versucht werden, mithilfe der Wahrscheinlichkeitstheorie, etwa durch Anwendung des Bayes'schen Theorems, in Kombination mit prospektiven Studien an grossen Patientenkollektiven, die Wahrscheinlichkeitsraten des Auftretens bestimmter Symptome quantitativ zu bestimmen - wie dies die holländische Gruppe um Lex Rutten [17] kürzlich für sechs Symptome des Repertoriums durchgeführt hat, oder Heiner Frei bei der Polaritätsanalyse von Symptomen aus Bönnighausens Therapeutischem Taschenbuch [18]. Damit wären aber diejenigen Aspekte der Materia medica, die sich mit streng mathematisch-naturwissenschaftlichen Methoden bearbeiten liessen, weitgehend erschöpft. Der Rest, d.h. was die Materia medica sonst noch an Information enthält, wäre - aus streng mathematisch-naturwissenschaftlicher Sicht - wertlos.

Was auf diese Weise in einzelnen Fällen als Fortschritt erscheinen und eine messbare Effektivitätssteigerung der Homöopathie bewirken mag, wäre in der theoretischen Verallgemeinerung dieses Ansatzes jedoch fatal. Dürfte man in der Homöopathie nichts anderes gelten lassen als das, wofür es externe mathematisch-naturwissenschaftliche Evidenz gibt, würde der gesammelte Reichtum des jahrhundertealten Corpus des homöopathischen Arzneischatzes auf einige Messwerte und Prozentzahlen zusammenschnurren. Die Praxis der Homöopathie lebt aber wesentlich von dem, was über diese Form reduktionistischer Datenanalyse hinausgeht.

Beanspruchen Homöopathen allerdings, auch in dem Bereich ihrer Methodik wissenschaftlich vorzugehen, der von der quantifizierenden Naturwissenschaft nicht mehr gedeckt ist, und soll dies im rationalen Diskurs mit Nachbardisziplinen oder politischen Entscheidungsträgern auch wahrgenommen und anerkannt werden, bedarf es dazu einer gesonderten Legitimation - etwa durch eine gute eigene Theorie. Wie die Erfahrung lehrt und wie sich auch wissenschaftssoziologisch und medizinhistorisch zeigen lässt, reichte der Hinweis auf die praktische Wirksamkeit einer Heilmethode nie zu deren Anerkennung aus. Der Durchbruch eines neuen Paradigmas kam (fast) immer erst dann zustande, wenn - und zwar mit oder ohne anfängliche Wirksamkeit - es eine plausible Theorie gab, die entweder den vermeintlichen Wirkmechanismus erklärte oder zumindest an positiv konnotierte zeitgenössische Vorstellungen anknüpfte [19].

Mit Hahnemanns Theorien $\mathrm{zu}$ argumentieren, erscheint unter den heutigen Bedingungen postmoderner Ideologiekritik nicht sinnvoll, da diese weder wissenschaftlich haltbar noch anschlussfähig an einen laufenden akademischen Diskurs sind. Schon Constantin Hering hatte sich - als strikter Hahnemannianer - von jeder theoretischen Erklärung Hahnemanns distanziert [20]. Die Idee einer Lebenskraft etwa, die - durch externe Arzneireize veranlasst - automatisch Symptome produziere, die von einem vorurteilslosen Beobachter objektiv registriert, aufgelistet und zu einer Materia medica zusammengefasst werden, war zu Hahnemanns Zeit noch eine in weiten Teilen der Ärzteschaft durchaus plausible und kommunizierbare Theorie.

Aus heutiger neurophysiologischer, kognitionswissenschaftlicher und erkenntnistheoretischer Sicht erweist sich diese theoretisch-naive Vorstellung jedoch in dem Sinne als dekonstruierbar, dass weder eine Lebenskraft noch Arzneireize noch Symptome als ontologische Entitäten voneinander isolierbar noch diese durch einen trivialen Automatismus verknüpft seien und es weder einen vorurteilslosen Beobachter noch eine objektive Wahrnehmung, geschweige denn Bewertung von Symptomen gebe. Bei deren Erfassung handle es sich vielmehr stets um einen hochkomplexen semiotisch-hermeneutischen Prozess, der nicht nur vom individuellen Beobachter und dessen $\mathrm{Ge}$ schichte, sondern auch kontext- und kulturabhängig sei $[21,22]$, was dem Konzept einer «Reinen Arzneimittellehre», die ja nur objektive Fakten enthalten soll, den Boden entziehe.

Wer aber den aus methodischem Reduktionismus bestehenden sicheren Hafen der klassischen Naturwissenschaften verlässt, um sich - in der Hoffnung auf theoretische Unterstützung - nach Allianzen mit den modernen Geistes- und Sozialwissenschaften umzusehen, findet sich bald auf hoher See und muss zusehen, wie ihm die eigenen theoretischen Grundlagen unter den Fingern zerrinnen. Als ebenso wenig geeignet, ein Argument für die Wissenschaftlichkeit der homöopathischen Arzneimittelprüfung und Praxis abzugeben, erweist sich die moderne Quantenphysik. Zum einen wird diese kaum von den Physikern selbst, geschweige denn von Medizinern verstanden (von dem renommierten Quantenphysiker Richard Feynman (1918-1988) stammt der Satz: «I think I can safely say nobody understands quantum mechanics!») [23]. Zum anderen gelten die Phänomene des Welle-Teilchen-Dualismus 
und der Unschärferelation nur im Mikrobereich von Elektronen und Photonen, während sie sich bereits auf Molekülebene durch Dekohärenz bzw. Wechselwirkung mit der Umgebung ausmitteln, weshalb ab da, d.h. im Bereich der Medizin und Pharmakologie, die klassische Newton'sche Physik gelte [24].

Wenn auch aus der Quantentheorie kein direktes Argument für die Wissenschaftlichkeit der Materia medica homoeopathica zu machen ist, so lässt sich indirekt an ihr doch etwas bemerken, was der Theorie der Homöopathie ebenso wie der Medizintheorie im Allgemeinen zugutekommen könnte. Die theoretische Physik hat es spätestens seit der Kopenhagener Erklärung 1926 aufgegeben, zu behaupten, sie könne so etwas wie die «Wahrheit» der Welt ergründen. Sie könne zwar aufgrund von Modellen sehr genaue Voraussagen machen und diese durch Messungen überprüfen. So können - je nach Versuchsaufbau - beim Doppelspalt-Experiment an dem sich dahinter befindlichen Schirm entweder Teilchen oder Wellen gemessen werden, aber nicht beides zugleich. Auf diese Weise könne aber niemals entschieden werden, was nun «wirklich» durch den Doppelspalt ging: Wellen oder Teilchen? Die Naturwissenschaft als solche hat damit kein Problem. Sie zieht sich auf den rein instrumentellen Charakter ihrer Theorien und Modelle zurück und hat damit Erfolg. Ob die Welt in «Wahrheit» aus Wellen oder Teilchen besteht - diese Frage zu enträtseln, überlässt die Physik den Philosophen $[25,26]$.

Die Homöopathie könnte sich nun gleichermassen ein derart instrumentelles Verständnis ihrer Maximen und Theorien zu eigen machen. Immerhin sind das «Organon» und die Theorien Hahnemanns (sowie seiner Nachfolger) als «Werkzeuge» des echten Heilkünstlers konzipiert. Konsequent zu Ende gedacht, hiesse das aber, auf den Anspruch Hahnemanns (und seiner Anhänger), mit der Homöopathie die «Wahrheit» bzw. den «einzig richtigen» und «möglichen» «Heilweg» [27] erkannt zu haben, zu verzichten.

An dieser Stelle gerät das Ringen um eine zeitgemässe Theorie der Homöopathie bzw. eine Medizintheorie, will sie auch dies noch - samt dem dazugehörigen Horizont erfassen, an die eigenen Grenzen. Das Problem, das Verhältnis der Homöopathie zur Naturwissenschaft zu bestimmen, nähert sich - ausgehend von dessen Relevanz für ein konkretes Projekt (Revision der Materia medica) über die Grundsatzfrage, ob bzw. inwieweit dieses überhaupt wissenschaftlich durchführbar sei - nun dem Subjekt der Fragestellung, also den einzelnen Homöopathen, die von dem Willen beseelt sind, ebendies zu vollbringen! Was berechtigt sie zu ihrer Zuversicht? Stillschweigend vorausgesetzt wird immerhin, dass erstens der Homöopathie nichts Besseres passieren könne, als wissenschaftlich bearbeitet $\mathrm{zu}$ werden, und man zweitens dadurch der Wahrheit näher käme. Dass diese - unhinterfragten - Voraus- setzungen nicht selbstverständlich sind, sondern vom jeweils vorherrschenden Zeitgeist abhängen, mag ein Exkurs in die europäische Kulturgeschichte zeigen.

\section{Wissenschaftshistorischer Hintergrund}

Für Hippokrates, den antiken Stammvater der Ärzte, war Medizin eine Heilkunst (téchne iatriké). In der klassischen Schrift «perì tópon tón kát” ánthropon» («Über die Stellen des Menschen»), aus der auch Hahnemann im «Organon» zitiert [28], geht der Verfasser sogar so weit, zu behaupten, dass es in der Medizin überhaupt keine Regel geben könne, die immer ohne Ausnahme gelte (weder das Simile-Prinzip noch das Contrarium-Prinzip noch das Prinzip des «mal dies, mal das»), da alles auf den Einzelfall im jeweiligen Kontext ankomme, ja sogar auf den einmaligen Moment, griechisch «kairós». Prinzipien und Theorien haben demnach nur instrumentellen Charakter, und ihre kunstgemässe Auswahl und Anwendung beim individuellen Patienten obliege der Beurteilung des Heilkünstlers [29]. Diese Tradition des Primats der Heilkunst über alle Systeme, Dogmen, Spekulationen sowie Regeln und Prinzipien lässt sich durch die gesamte Medizingeschichte verfolgen - von Hippokrates über Aretaeus und Sydenham bis zu Hahnemann, wie Letzterer auch selbst bemerkte [30].

Gleichwohl ist diese Linie der Heilkunst in der Historiographie mehr als unterrepräsentiert. Seit jeher werden vorwiegend diejenigen Mediziner in der Literatur verewigt, die durch die Aufstellung von besonderen Lehrsätzen und Systemen, nicht unbedingt durch gute Praxis, hervorgestochen sind. (Ein ähnliches Phänomen findet sich in der Religionsgeschichte als Dogmengeschichte, Kirchengeschichte, Geschichte der Päpste usw.) Um diese kollektive Bevorzugung allgemeiner, abstrakter, vermeintlich wissenschaftlicher «Wahrheiten» $\mathrm{zu}$ verstehen, muss man noch einen weiteren Schritt zurücktreten und sich vergegenwärtigen, welch folgenreicher Wertewandel in der griechischen Antike mit der Erfindung der Wissenschaft (epistéme) stattgefunden hat.

Gemäss der Analyse Friedrich Nietzsches (1844-1900), der die Wurzeln des europäischen Nihilismus in eben dieser Zeit verortet, waren es Sokrates und Platon, die durch die Einführung des Begriffs «Wahrheit» die ursprüngliche Wertschätzung des realen Lebens, der Kunst und der Praxis unterminierten - um die Menschen zum Glauben an eine ideelle (sozusagen virtuelle) Welt des Wahren, Guten und Gerechten zu überreden. Das in diesem Umkreis entstandene Ideal der Wissenschaft bestand demgemäss in (vermeintlich) «wahren», weil abstrakten, logischen und vor allem beweisbaren Sätzen bzw. Systemen - eine Definition, die für die praktische situative Intelligenz, z.B. eines Heilkünstlers, keinen Raum mehr liess. 
Nun ist aber das, was sich beweisen lässt, oft wenig wert. Es kann sogar eher Misstrauen erzeugen, wenn man zum einen bedenkt, welch geringer Teil des Lebens überhaupt beweisbar ist, und zum anderen, welche Manipulationsund Suggestionskraft sog. wissenschaftliche Beweise haben (wie jeder Demagoge weiss). Nach Nietzsches Einschätzung besteht das Wesen der Wissenschaft gerade darin, «vieles niemals $(\mathrm{zu})$ sehen, vieles falsch $(\mathrm{zu})$ sehen, vieles hinzu(zu)sehen» [31]. Ganz im Gegensatz zur üblichen, gewohnten Lesart wird hier der Anspruch auf Wahrheit, Wissenschaft und Beweisbarkeit geradezu als Ursache wie auch als Folgeerscheinung der abendländischen «Decadence» interpretiert.

Man muss freilich nicht so weit gehen wie Nietzsche, der in «unserer Wissenschaft» eine "Krankheits-Form» sieht [32], doch stellt sich vor dem Hintergrund einer so fundamentalen Kritik der europäischen Wissenschaftskultur die Frage des Verhältnisses zwischen Heilkunst und Wissenschaft in radikalisierter Form. Immerhin hat man es bei dem Idol Wissenschaft - erst recht seit der sog. wissenschaftlichen Revolution im 17. Jahrhundert [33] - mit einer Art Mega-Trend der Neuzeit zu tun, d.h. mit einer über die letzten Jahrhunderte immer mächtiger gewordenen Determinanten, die alle Bereiche unseres Lebens durchdringt und bestimmt.

Spätestens seit der Säkularisation hat der Glaube an die Wissenschaft, speziell an die Naturwissenschaften, geradezu religiöse Züge angenommen, wie sich nicht nur an der kultischen Verehrung Auguste Comtes (1798-1857), des Begründers des Positivismus, über dessen Tod hinaus zeigt. Nach dem Niedergang des konfessionellen christlichen Glaubens blieb in der Moderne keine höhere Autorität und Richtinstanz übrig als die Wissenschaft. Und so wird heute - in Ermangelung anderer Werte und Kriterien - im Zweifelsfall alles wissenschaftlich untersucht, verhandelt und entschieden - von der Erziehung, Bildung, Jurisprudenz, Wirtschaft und Politik bis hin zur Medizin.

Bereits zu Hahnemanns Zeit war der Druck, der von den technischen Erfolgen der Naturwissenschaften auf die Medizin ausging, massiv zu spüren. Im Zuge der Frühindustrialisierung war das Geld gerade dabei, zum neuen Subjekt der Geschichte zu werden und alles andere, also Gesellschaft, Wirtschaft, Wissenschaft, Kultur und Politik, zu seinem Prädikat oder Attribut zu degradieren [34]. Die damit einhergehende Entfremdung der Menschen von ihrer Tätigkeit und ihren Werken sowie die zunehmende Komplexität des Lebens disponierte sie unter anderem dazu, mehr als je zuvor ihr Heil in abstrakten Begriffen wie Aufklärung, Autonomie, Emanzipation und Wissenschaft statt in der gelungenen eigenen Lebenspraxis zu suchen.

Und so fühlte sich die gesamte Medizinergeneration Hahnemanns (einschliesslich der Philosophen, wie Schelling und andere) förmlich getrieben, die Medizin in den Rang einer Wissenschaft zu erheben, ohne freilich die sozioökonomischen Wurzeln dieses Drucks von Seiten des Zeitgeistes zu ahnen. Dementsprechend heftig und profund wurde zu jener Zeit noch einmal abschliessend das Thema diskutiert, ob Medizin Heilkunst oder Heilkunde sei [35] - bis es dann für die nächsten zwei Jahrhunderte ausgemacht erschien, dass Medizin angewandte Naturwissenschaft sei.

Auch Hahnemann, obgleich Sohn eines Porzellanmalers und selbst begnadeter Heilkünstler, war - mindestens bis 1810 - von diesem Sog erfasst und zollte ihm Tribut, was sich unter anderem im Titel der 1 . Auflage des «Organon» niederschlug [36]. Ab der 2. Auflage (1819) benannte er es um in "Organon der Heilkunst» [37], was als Rückbesinnung auf die eigentliche Berufung des Arztes als Heilkünstler gedeutet werden kann [38]. Dieser Schritt Hahnemanns muss keineswegs als Wissenschaftsfeindlichkeit verstanden werden, zumal er bis zuletzt am Ideal der Wissenschaft festhielt. Offensichtlich hatte Hahnemann aber schlechte Erfahrungen mit zeitgenössischen Kollegen gemacht, die den Begriff «rationelle Heilkunde» (und damit das Konzept der Medizin als Wissenschaft) diskreditierten, sodass dieser - als abstrakter Begriff bzw. als Identifikations-Label - für ihn untragbar wurde.

So schliesst sich auch der Kreis zu Nietzsches verheerender Kulturkritik an Begriffen wie Wahrheit, Moral oder Wissenschaft. Bei näherem Licht betrachtet, hatte Nietzsche nichts gegen Begriffe wie "Gott», "Wahrheit» oder "Weisheit» als solche (immerhin hat er sie selbst verwendet), solange sie nur nicht von unwürdigen sog. «Muckern» in deren flachem Sinne uminterpretiert und damit verfälscht würden [39]. Haben sich allerdings real existierende Wissenschaft und Metaphysik erst einmal so weit vom wirklichen Leben entfernt und zu Weltbildern verselbstständigt, dass sie sozusagen kontraproduktiv werden und dieses behindern, anstatt unterstützen, muss der Mensch zum «Künstler» werden, um wieder «Herr über den Stoff, Herr über die Wahrheit» sein zu können [40].

\section{Medizintheoretische Konsequenzen}

In Analogie hierzu lässt sich die eingangs gestellte Frage nach der Medizintheorie nun so beantworten, dass die Medizin, und erst recht die Homöopathie, gut daran täten, bei einer Wesensbestimmung ihrer selbst die Dimension der Heilkunst höher zu gewichten (Hierarchisierung) als den heute unabweisbaren Impuls zu einer Identifizierung mit der Wissenschaft. Der Anspruch auf Wissenschaftlichkeit der Medizin klingt sicher mächtig und respektabel. Da jedoch alles davon abhängt, welche Art von Wissenschaft zu welchem Zweck unter welchen Umständen auf welches Einzelproblem angewandt wird, bedarf es immer auch der 
Kunst der Betrachtung, Beurteilung und Bewertung. Ebendiese genuin menschlichen Handlungen kann aber - verantwortungsvoll - nur ein Heilkünstler vollbringen, der die verschiedenen wissenschaftlichen Ansätze und Methoden souverän beherrscht und dem jeweiligen Gegenstand entsprechend einsetzt - sei es bei der Behandlung von $\mathrm{Pa}-$ tienten oder der Revision der Materia medica.

Mit dieser Position wäre die Homöopathie auch für die Herausforderungen der Postmoderne gerüstet. Sie könnte den - postmodern unhaltbaren - absoluten Wahrheitsanspruch Hahnemanns im Bewusstsein aufgeben, dass wissenschaftliche Theorien und Methoden stets Werkzeugcharakter besitzen und es dem Heilkünstler obliegt, je nach Zweck und Umständen die eine oder andere als die angemessenste und damit (relativ) wahre zu qualifizieren. Auch die - postmodern unhaltbaren - metaphysischen Begründungen der Homöopathie durch Hahnemann, wie Gott, Vorsehung oder Teleologie [41], wären entbehrlich, indem es dem von der absoluten Last dieser ideologischen Begriffe entbundenen Heilkünstler freistünde, diese - für sein persönliches Weltbild - je nach Kontext in neuer Bedeutung zu gebrauchen oder auf sie zu verzichten.

Durch die Überwindung bzw. Relativierung dieser Relikte alten dogmatischen Denkens könnte andererseits der funktionelle Praxis- und Kunstaspekt der Homöopathie zur Geltung kommen. Wahrheit wird postmodern ja nicht ganz negiert. Doch Wahrheit gibt es nur in Perspektive. Und diese ist bei jedem Patienten eine andere. Gerade die Herausforderungen postmoderner Polyperspektivität könnte die Homöopathie aber gut parieren, indem sie pragmatisch, instrumentell und ohne ideologische Vorbedingungen ein semiotisches Kombinationssystem sowie einen Arzneischatz von Zeichen und Symptomen zur Verfügung hält, der - ganz im Sinne echter Heilkunst - über genug Flexibilität und Schärfe verfügt, um jeden erdenklichen singulären Fall therapeutisch abzubilden.

Auf diesen Kunstcharakter der Homöopathie müsste auch bei dem Projekt der Revision der Materia medica homoeopathica geachtet werden. Anders als bei einem Primat naturwissenschaftlicher, quantitativer oder evidenzbasierter Ambitionen und einer daraus resultierenden Verführbarkeit durch das technisch Machbare müsste der oberste Wert die Praxistauglichkeit, d.h. sowohl die Angemessenheit an den Heilzweck als auch die Handhabbarkeit des Produkts, sein. Was für einen Naturwissenschaftler, Informatiker oder Statistiker das Höchste wäre, muss es für den Heilkünstler noch lange nicht sein. Schon zur Abwendung einer drohenden Deprofessionalisierung [42] dürften sich Heilkünstler nicht von externen (ideologischen oder ökonomischen) Interessen fremdbestimmen lassen.

Anstatt, wie aus einer wissenschaftsnaiven Sicht vielleicht naheliegend, Vollständigkeit, Einheitlichkeit und philologische Exaktheit als oberste Prioritäten anzustreben, wäre dem Heilkünstler möglicherweise mehr damit gedient, statt einer Einheitsdatenbank von wörtlichen Zitaten z.B. mehrere kleinere, verdichtete, methodisch verschieden aufgebaute, dafür aber in sich stimmige Repertorien zu erstellen. Und statt sich Karl Poppers Wissenschaftskriterium der Falsifikation, das wissenschaftstheoretisch längst widerlegt ist [43] und nicht einmal in den härtesten Naturwissenschaften funktioniert [44], zum Vorbild zu nehmen und gutgläubig ausgerechnet zur Revision der Materia medica homoeopathica einzusetzen, würde ein Heilkünstler, der den Primat der Kunst über eine fragliche Wissenschaftlichkeit verinnerlicht hat, eher darauf achten, den Arzneischatz nicht - durch Aufsitzen auf fachfremde Philosopheme - unnötig zu dezimieren.

Anhand dieser wenigen Beispiele mag man erkennen, wie wichtig es sein könnte, vor grösseren Projekten selbstkritisch die eigene Position, die Rahmenbedingungen sowie Sinn und Zweck des ganzen Unternehmens zu reflektieren. Je nach theoretischen Prämissen könnte das Ergebnis sehr unterschiedlich ausfallen.

Die hier vorgeschlagene und begründete Position, die Homöopathie primär als Heilkunst und erst sekundär als Wissenschaft zu konzipieren und zu praktizieren, belässt der Wissenschaft und der sich als Naturwissenschaft verstehenden Hochschulmedizin alle Rechte als solcher. Sie verwahrt sich lediglich gegen Übergriffe derselben und Ansprüche der Art, dass Wert und Praxis der Homöopathie ausschliesslich am Mass der Naturwissenschaft zu messen seien. Im Zweifelsfall würde vielmehr die Heilkunst, nicht die Wissenschaft, das letzte Wort haben.

Der Unterschied der hier vertretenen Position zum dominierenden, zeitgeistbedingten absoluten Wissenschaftsoptimismus besteht allein in einer anderen Priorisierung von Kunst und Wissenschaft. Ein minimaler Unterschied in der Hierarchisation, der von konventionellen Wissenschaftlern womöglich gar nicht wahrgenommen wird (weil ihnen die Kategorien dazu fehlen), könnte aber enorme, nicht zu unterschätzende Auswirkungen haben - was Homöopathen eigentlich vertraut sein müsste.

Medizintheorie stellt also - im Geist eines Heilkünstlers betrieben - keinen Gegensatz zur Praxis dar, sondern lässt sich (wie schon Hahnemann wusste) als ein Werkzeug zur Vervollkommnung der Heilkunst begreifen und einsetzen. Dass an jedem Werkzeug weiter gefeilt und gearbeitet werden kann, versteht sich. Dass es aber ohne scharfe Werkzeuge (einschliesslich Werkzeugmacher) nicht geht, versteht sich ebenfalls. Insofern gilt wohl nach wie vor der von Kurt Lewin (1890-1947) geprägte Satz: «Es gibt nichts Praktischeres als eine gute Theorie» [45].

\section{Disclosure Statement}

The author declares that no financial or other conflict of interests exists in relation to the content of this article. 


\section{Literatur}

1 Schmidt JM, Kaiser D (Hrsg): Samuel Hahnemann. Gesammelte kleine Schriften. Heidelberg, Haug, 2001, p 390.

2 Schmidt JM (Hrsg): Samuel Hahnemann. Organon der Heilkunst, ed 6 (1842). Textkritische Ausgabe. Heidelberg, Haug, 1992.

3 Hahnemann S: Die chronischen Krankheiten, ihre eigenthümliche Natur und homöopathische Heilung. 1. Theil, 2. Auflage. Dresden und Leipzig, Arnold, 1835.

4 Schmidt JM: Hahnemann's legacy in a new light - a systematic approach to the Organon of Medicine. Homeopathy 2005;94:202-206.

5 Wischner M, Lucae C (Hrsg): Samuel Hahnemann. Gesamte Arzneimittellehre, 3 Bände. Stuttgart, Haug, 2007.

6 Schmidt JM (Hrsg): Samuel Hahnemann. Organon der Heilkunst, ed 6 (1842). Standardausgabe. Heidelberg, Haug, 1996, \$ 143 und $\$ 144$.

7 Wieland W: Die Diagnose. Überlegungen zur Medizintheorie. Berlin, de Gruyter, 1975. Neuauflage: Warendorf, Hoof, 2004

8 Wiesing U: Wer heilt, hat Recht? Über Pragmatik und Pluralität in der Medizin. Stuttgart, Schattauer, 2004

9 Aristoteles: Metaphysik. Buch A. 981a.

10 Schmidt JM: Die Homöopathie im Spannungsfeld zwischen aristotelischer und neuzeitlicher Wissenschaft. Schweiz Zschr Ganzheitsmedizin 2009;21:105-111.

11 Wischner M: Ähnlichkeit in der Medizin. Über die Wissenschaftlichkeit von Homöopathie und Schulmedizin. Essen, KVC, 2004.

12 Schmidt JM: Die Entstehung, Verbreitung und Entwicklung von Heilsystemen als Gegenstand der Medizingeschichte - am Beispiel der Homöopathie. Sudhoffs Arch 2007;91:3872 .

13 Fräntzki E: Die Idee der Wissenschaft bei Samuel Hahnemann. Heidelberg, Haug, 1976.

14 Schmidt JM, Kaiser D (Hrsg): Samuel Hahnemann. Gesammelte kleine Schriften. Heidelberg, Haug, 2001, pp 375, 390, 500.

15 Luft B, Wischner M (Hrsg): Samuel Hahnemann. Organon-Synopse. Heidelberg, Haug, 2001, p 559 (\$ 145).

16 Habich K, Köster C, Rohwer J (eds): A step forward that makes sense - or a step back to the days before Hahnemann? An international discussion about modern trends, diversity of methods, and genuine homoeopathy. www.grundlagen-praxis.de (Grundlagendebatte).

17 Rutten L, Stolper E, Lugten, R, Barthels R: Updating the Repertory: Do It the Bayesian Way. Abstract Book of the 64th Congress of the Liga Medicorum Homeopathica Internationalis. August 26-30, 2009. Warsaw, 2009, p 58.

18 Frei H: Effiziente homöopathische Behandlung. Ein strukturiertes Konzept für den Praxisalltag. Stuttgart, Haug, 2008.

19 Unschuld P: Was ist Medizin? Westliche und östliche Wege der Heilkunst. München, Beck, 2003.

20 Schmidt JM: Taschenatlas Homöopathie in Wort und Bild. Grundlagen, Methodik und Geschichte. Heidelberg, Haug, 2001, p 75.

21 Walach H: Psychologie. Wissenschaftstheorie, philosophische Grundlagen und Geschichte. Stuttgart, Kohlhammer, 2005.

22 Bauer AW: Axiome des systematischen Erkenntnisgewinns in der Medizin. Der Internist 1997;38:299-306

23 Feynman R: The Character of Physical Law. Cambridge, MA, MIT Press, 1967, Chapter 6.

24 Lesch H: Quantenmechanik für die Westentasche. München, Piper, 2007.

25 Scheibe E: Die Philosophie der Physiker. München, Beck, 2006.

26 Atmanspacher H: Quantenphilosophie. Zum 100. Geburtstag der Quantentheorie. Neue Zürcher Zeitung 13.12.2000;291:73.

27 Schmidt JM: Samuel Hahnemann. Organon der Heilkunst, ed 6 (1842). Neuausgabe. Heidelberg, Haug, 1999, $\$ 109$ und $\$ 53$.

28 Schmidt JM (Hrsg): Samuel Hahnemann. Organon der Heilkunst, ed 6 (1842). Neufassung München, Elsevier, 2003, p 45

29 Hippokrates: Des lieux dans l'homme; in Littré É (ed): Euvres complètes d'Hippocrate. Paris, Baillière, 1849, Band 6, pp 276-349.

30 Schmidt JM, Kaiser D: Samuel Hahnemann. Gesammelte kleine Schriften. Heidelberg, Haug, 2001, p 34.

31 Nietzsche F: Nachgelassene Fragmente. November 1887-März 1888; in Colli G,
Montinari M (Hrsg): Friedrich Nietzsche. Sämtliche Werke. Kritische Studienausgabe. München, dtv, 1980, Band 13, p 193.

32 Nietzsche F: Nachgelassene Fragmente Frühjahr-Sommer 1888; in Colli G, Montinari M (Hrsg): Friedrich Nietzsche. Sämtliche Werke. Kritische Studienausgabe. München, dtv, 1980, Band 13, p 504.

33 Shapin S: The Scientific Revolution. Chicago, University of Chicago Press, 1996

34 Brodbeck KH: Die Herrschaft des Geldes. Geschichte und Systematik. Darmstadt, Wissenschaftliche Buchgesellschaft, 2009.

35 Wiesing U: Kunst oder Wissenschaft? Konzeptionen der Medizin in der deutschen Romantik. Stuttgart, frommann-holzboog, 1995.

36 Hahnemann S: Organon der rationellen Heilkunde. Dresden, Arnold, 1810.

37 Hahnemann S: Organon der Heilkunst, ed 2. Dresden, Arnold, 1819

38 Resch G, Gutmann V: Wissenschaftliche Grundlagen der Homöopathie. Berg, O.-Verlag, 1986.

39 Nietzsche F: Nachgelassene Fragmente. Herbst 1887; in Colli G, Montinari M (Hrsg): Friedrich Nietzsche. Sämtliche Werke. Kritische Studienausgabe. München, dtv, 1980, Band 12, pp 579-580.

40 Nietzsche F: Nachgelassene Fragmente. November 1887-März 1888; in Colli G, Montinari M (Hrsg): Friedrich Nietzsche. Sämtliche Werke. Kritische Studienausgabe. München, dtv, 1980; Band 13, p 194.

41 Schmidt JM: Die philosophischen Vorstellungen Samuel Hahnemanns bei der Begründung der Homöopathie. München, Sonntag, 1990

42 Unschuld PU: Der Arzt als Fremdling in der Medizin? München, Zuckschwerdt, 2005.

43 Kuhn TS: The Structure of Scientific Revolutions. Chicago, University of Chicago Press, 1962.

44 Feyerabend P: Against Method. Outline of an Anarchistic Theory of Knowledge. London, Verso, 1975.

45 Lewin K: Field theory and learning; in Cartwright D (ed): Field Theory in Social Science: Selected Theoretical Papers. New York, Harper \& Row, 1951, p 169. 